\title{
Psicologia Cognitiva e Consumo: O Impacto da Quantidade de Informações na Configuração de Objetivos
}

\author{
Cognitive Psychology and Consumption: The Impact of the Amount \\ of Information on Goal Framing
}

\author{
Leandro Miletto Tonetto, ${ }^{*}$ \& Lilian Milnitsky Stein ${ }^{b}$ \\ ${ }^{a}$ Universidade do Vale do Rio dos Sinos, Porto Alegre, Brasil. \\ $\&{ }^{b}$ Pontifícia Universidade Católica do Rio Grande do Sul, Porto Alegre, Brasil
}

\begin{abstract}
Resumo
O efeito de configuração de objetivos refere-se ao achado de que distintas formas de apresentação de um mesmo conteúdo de comunicação tendem a gerar diferentes graus de persuasão nos consumidores. Esse resultado parece um reflexo de respostas automáticas (intuitivas) dadas pelo consumidor frente a menores quantidades de informação. Este artigo apresenta os resultados de um experimento que investigou o impacto da quantidade de informações no efeito de configuração de objetivos. Participaram do experimento 296 estudantes universitários, designados a quatro grupos constituídos em função das variáveis configuração (ganhos e perdas) e quantidade de informações (expandidas e resumidas). Os resultados evidenciaram um efeito de configuração apenas entre as versões resumidas do texto, sendo a configuração de ganhos considerada a mais convincente.

Palavras-chave: Psicologia do consumidor, julgamento e tomada de decisão, racionalidade limitada, efeito de configuração de objetivos, quantidade de informações.
\end{abstract}

\begin{abstract}
Goal framing effect refers to the finding that different ways of presenting the same content of communication tend to generate different degrees of persuasion among consumers. It seems to be a reflex from automatic (intuitive) responses given by consumers regarding low amounts of information. This research shows the results of an experiment investigating the impact of the amount of information on goal framing effect. In a between-subject design experiment, 296 undergraduates were split in four conditions, being exposed to different text frames (gains and losses) and amounts of information (extended and abbreviated). The results have shown goal framing effect only for the abbreviated versions of the text, being gain frame the most persuasive.

Keywords: Consumer psychology, judgment and decision making, bounded rationality, goal framing effect, amount of information.
\end{abstract}

Que tipo de linguagem produz maiores níveis de persuasão na comunicação com os consumidores? Deve-se enfatizar o ganho esperado com o uso de um produto ou a perda iminente proveniente da utilização de produtos concorrentes? Estas questões se fazem presentes no cotidiano dos profissionais de comunicação e marketing, podendo ser relacionadas ao achado de que as pessoas respondem de formas distintas a diferentes apresentações de um problema decisório, fenômeno denominado por Tversky e Kahneman (1981) como efeito de configuração.

Os estudos sobre o efeito de configuração, no entanto, não surgiram no contexto da comunicação social. Essas

\footnotetext{
*Endereço para correspondência: Universidade do Vale do Rio dos Sinos, Rua Luiz Manoel Gonzaga, 744, Três Figueiras, Porto Alegre, RS, Brasil 90470-280. E-mail: ltonetto@yahoo.com e lilian@pucrs.br.

Esta pesquisa teve apoio da Coordenação de Aperfeiçoamento de Pessos de Nível Superior (CAPES).
}

pesquisas surgiram inicialmente em estudos como os de Tversky e Kahneman (1981), na área da Psicologia Cognitiva, em um dilema relacionado a uma doença asiática hipotética. Nesse dilema, as pessoas conheciam os riscos envolvidos em cada possível escolha de tratamento para a doença, caracterizando o que se pode denominar como tomada de decisão sob risco. Além disso, os problemas foram configurados de forma a ressaltar ganhos ou perdas advindos dos programas de tratamento, o que modificou as escolhas das pessoas em função da variação das formas de apresentação de informações sobre a tarefa decisória (para uma revisão, ver Tversky \& Kahneman, 1981).

A partir do estudo pioneiro de Tversky e Kahneman (1981), uma série de pesquisas sobre o efeito de configuração passou a ser desenvolvida por pesquisadores de todo o mundo (Almashat, Ayotte, Edelstein, \& Margrett, 2009; Bettman, Luce, \& Payne, 1998; Fagley \& Miller, 1997; Ganzach \& Karsahi, 1995; Ganzach, Weber, \& Bem Or, 
1997; Kühberger, 1995; LeBoeuf \& Shafir, 2003; Mellers, Schwartz, \& Cooke, 1998; Payne, Bettman, Coupey, \& Johnson, 1992, entre outras). Diversas áreas, como saúde, segurança e marketing, foram beneficiadas por essas pesquisas, mesmo que os resultados nem sempre tenham se mostrado consistentes.

Além disso, a literatura apresenta dados discordantes no que se refere a qual configuração (ganhos ou perdas) seria a mais persuasiva sobre as pessoas (Levin, Gaeth, Schneider, \& Lauriola, 2002; Levin, Schneider, \& Gaeth, 1998). Essas divergências parecem se dever ao fato de que o efeito de configuração não é apenas estudado no contexto das decisões sob risco, a exemplo do dilema da Doença Asiática. Diversas decisões do dia-a-dia podem ser caracterizadas como decisões de incerteza, pois as pessoas desconhecem riscos associados a cada possível resultado, como "Qual candidato votarei nas próximas eleições?" ou "Qual cartão de crédito devo utilizar?". As novas abordagens ao efeito de configuração, segundo estes autores, parecem ter levado também à identificação de padrões divergentes de respostas frente a diferentes configurações dos problemas decisórios.

Partindo desta proposta, Levin et al. (1998) propuseram que há três diferentes tipos de efeitos de configuração presentes na literatura: o efeito de configuração sob risco, de atributos e de objetivos. Diferenças na definição do que se compreende pelo termo configuração podem, nesse sentido, refletir em modificações metodológicas que desencadeariam mudanças na forma com que os sujeitos processariam informações e tomariam decisões.

$\mathrm{O}$ efeito de configuração sob risco, introduzido por Tversky e Kahneman (1981), pode ser ilustrado com o dilema da doença asiática e, conseqüentemente, com a disposição das pessoas para assumir riscos conhecidos. Já a configuração de atributos afeta a avaliação de características de objetos ou eventos na avaliação de atratividade de um item único, enquanto que a configuração de objetivos envolve o grau de persuasão de um conteúdo comunicado para ações ou comportamentos (Levin et al., 1998).

Os estudos de comunicação freqüentemente trabalham com o efeito de configuração de objetivos, por meio da manipulação semântica de diversos argumentos integrantes de uma mensagem. Ganzach e Karsahi (1995), por exemplo, desenvolveram estudos em cenário natural nos quais utilizaram como instrumentos duas versões de um texto promocional sobre cartões de crédito, configuradas em termos de ganhos e perdas. Nesse estudo, a versão de ganhos ressaltava as vantagens do uso de cartões de créditos, em comparação a outras formas de pagamento, enquanto que a versão de perdas enfatizava as desvantagens advindas do uso de outros métodos de pagamento em comparação à utilização de cartões de créditos. Para cada uma das configurações do instrumento, mensurou-se o grau de persuasão das mensagens sob os sujeitos, sendo a configuração de perdas a que evidenciou maior impacto. Os resultados apontaram que os consumidores foram mais receptivos a mensagens que salientavam perdas pelo não uso do cartão, que mensagens enfatizando os ganhos advindos de seu uso. Este efeito mais persuasivo da configuração de perdas persistiu em um follow-up seis meses depois.

Outro estudo sobre o efeito de configuração de objetivos foi desenvolvido por Ganzach et al. (1997). Os autores compararam o efeito de configuração de mensagens de comunicação em cenários artificial e natural. Para os integrantes do grupo de cenário artificial, as pessoas foram informadas que as questões a serem respondidas faziam parte de um experimento, enquanto que as pessoas integrantes do grupo de cenário natural foram instruídas a responder a essas questões considerando-as como uma situação da vida real. No cenário artificial, as pessoas demonstraram mais interesse em aderir à proposta do cartão na configuração de ganhos do que na configuração de perdas, enquanto que, no cenário natural, a configuração de perdas foi mais efetiva. A explicação para as divergências nos dados foi apontada como o envolvimento com a tarefa. Os autores sugeriram que, em cenário natural, as pessoas envolver-se-iam em um processamento da informação mais elaborado, enquanto que no cenário artificial se deixariam levar por informações periféricas.

Os achados de Ganzach e seus colaboradores (Ganzach \& Karsahi, 1995; Ganzach et al., 1997), sobre a maior persuasão produzida pelas versões de perdas em cenários naturais, representam o padrão de resultados esperados em estudos sobre o efeito de configuração de objetivos, diferentemente do que ocorre nas pesquisas sobre configuração sob risco (Levin et al., 1998). Quais seriam, então, as explicações para a existência do efeito de configuração de objetivos? Sendo o padrão de resultados destes estudos divergente em relação aos estudos clássicos de configuração sob risco, qual seria a abordagem teórica mais adequada para sua compreensão?

Quando o efeito de configuração de objetivos é detectado, os pesquisadores usualmente utilizam os postulados da Teoria Prospectiva (Tversky \& Kahneman, 1981) para explicá-los, tentando definir a situação experimental em termos de riscos implícitos que os participantes buscariam ou evitariam. No entanto, essas interpretações não parecem ser as mais adequadas, em função da dificuldade de identificação do risco relativo envolvido nas alternativas, quando se pesquisa o efeito de configuração de objetivos. Não seria possível, portanto, identificar quais alternativas seriam percebidas como de risco pelos participantes (Levin et al., 1998).

Meyerowitz e Chaiken (1987) ofereceram uma forma alternativa para interpretação do efeito de configuração de objetivos. Eles sugeriram a existência de um viés negativo no processamento da informação, ou seja, um impacto mais robusto da informação negativa que da positiva nos julgamentos. Haveria, ainda, a existência de um sistema para rápido reconhecimento de estímulos negativos, produzindo a diferença nos padrões de respostas encontrados (maior persuasão da informação negativa que da positiva sobre os sujeitos). 
Com base neste cenário, alguns argumentos adicionais podem ser delineados para compreensão do efeito de configuração de objetivos e, em especial, sobre o viés negativo no processamento das informações apresentadas em diferentes formas de configuração. Entre eles, destacam-se a percepção de risco dos consumidores (Finucane, Alhakami, Slovic, \& Johnson, 2000; Peters, Finucane, \& MacGregor, 2005), as interferências afetivas nos julgamentos (Peters et al., 2005; Slovic, Finucane, Peters, \& MacGregor, 2002), bem como o uso da intuição em tomada de decisão (Kahneman, 2003; Stanovich \& West, 2002).

Pode-se classificar a percepção de risco dos consumidores de duas formas distintas. O risco como análise refere-se a um processamento lógico, ao raciocínio e à deliberação científica sobre como lidar com dado problema, o que se faz presente em estudos de configuração sob risco, nos quais as pessoas conhecem as probabilidades associadas a cada alternativa disponível nos problemas decisórios. Já o risco como sentimento está relacionado a reações rápidas, instintivas e intuitivas em relação ao perigo (Finucane et al., 2000), e não, portanto, a dados concretos ou probabilidades conhecidas.

No que concerne às interferências afetivas nos julgamentos, entende-se que risco como sentimento estaria relacionado ao afeto. O risco é entendido como a qualidade específica de sentimentos experimentados como bons ou maus (de forma consciente ou não) e demarca qualidades positivas ou negativas dos estímulos (Peters et al., 2005). Segundo este argumento da interferência afetiva no julgamento, mesmo não envolvendo probabilidades associadas a cada possível resultado, como no efeito de configuração sob risco, a configuração de objetivos pode representar risco para as pessoas. Configurar objetivos salientando aspectos negativos denota perdas e, portanto, riscos.

Em marketing, o risco percebido está relacionado à confiabilidade da mensagem ou produto e ao envolvimento dos consumidores (Mitchell, 1999). Segundo Mittal (1995), o envolvimento seria o construto que se refere ao valor pessoal ou importância de algo para a pessoa, incluindo a relevância e a probabilidade de ocorrência dos eventos de risco.

Slovic et al. (2002) propuseram que, para lidar com contingências relacionadas ao risco, as pessoas utilizam comumente um mecanismo denominado heurística do afeto, que se refere ao uso dos sentimentos como informações para o processo decisório. A heurística do afeto entra em ação quando os sujeitos encontram-se em fase de avaliação de alternativas, ou seja, em processo de julgamento (Peters et al., 2005).

As imagens contidas na mente das pessoas são como "poças de afeto" (Peters et al., 2005), que contêm identificações positivas ou negativas, conscientes ou não. A intensidade dessas identificações varia com essas imagens. Ao realizarem seus julgamentos, as pessoas são influenciadas por esse afeto, ocasionando uma simpli- ficação no processamento da informação: trata-se da heurística do afeto em ação. Como exemplo, pode-se citar a concentração de produtos químicos nos alimentos, que tenderá a ser avaliada negativamente por pessoas contrárias ao uso de tais substâncias, independentemente de ser segura.

$\mathrm{O}$ afeto desempenha um papel central no julgamento e na tomada de decisão (Epstein, Lipson, Holstein, \& Huh, 1992). Proposições como essa levaram diversos autores a pesquisar as diferenças entre as decisões ditas racionais e as intuitivas e, mais recentemente, Kahneman (2003) enfatizou a importância da distinção entre intuição (mais emocional) e raciocínio (mais lógico), o que anteriormente Stanovich e West (2002) intitularam Sistema 1 e Sistema 2.

As operações do Sistema 1 seriam rápidas, automáticas, associativas, implícitas (não referentes à introspecção), emocionalmente carregadas, usualmente governadas pelos hábitos do sujeito, difíceis de controlar ou modificar e não requerem grandes esforços cognitivos. Já o Sistema 2 teria um funcionamento mais lento e serial (demandando maior esforço cognitivo), além de ser mais adequado para ser monitorado e controlado, apresentando um funcionamento relativamente flexível e potencialmente governado por regras (Kahneman, 2003).

O Sistema 1 opera de maneira similar aos processos perceptuais e não é limitado à estimulação presente. Os julgamentos intuitivos deparam-se com os conceitos e percepções da pessoa e podem ser evocados pela linguagem. Nesse modelo, o sistema perceptual e as operações intuitivas geram impressões acerca dos atributos de objetos percebidos ou imaginados, que não são nem voluntários nem verbalmente explícitos. Em contrapartida, os julgamentos são sempre intencionais e explícitos, mesmo quando não são expressos.

Já o Sistema 2 está envolvido com todos os julgamentos originados em raciocínio deliberado. O rótulo "intuitivo" é aplicado aos julgamentos que refletem diretamente impressões não modificadas pelo Sistema 2, já que uma das funções do Sistema 2 é monitorar a qualidade de operações mentais e comportamento manifesto (Kahneman, 2003).

Kahneman (2003), ao discutir as relações entre a proposta de existência dos Sistemas 1 e 2 e os efeitos de configuração, refere uma pesquisa de LeBoeuf e Shafir (2003). Em um único grupo, as pessoas foram solicitadas a responder diferentes questões com diversas formas de configuração. Usualmente, o que se esperaria é que estes sujeitos demonstrassem, por meio de suas decisões, os efeitos de configuração, mas os resultados de indivíduos mais reflexivos pareceram corroborar a sugestão de Kahneman (2003) em relação à existência de dois sistemas, em especial a do Sistema 2. As pessoas caracterizadas por um Sistema 2 mais ativo tiveram maior êxito em perceber relações entre duas formas de apresentação de um mesmo problema e em manter a consistência das respostas dadas a eles. 
Apesar do Sistema 1 possuir base afetiva, ambos - sistemas 1 e 2 - podem ser entendidos como racionais (Peters et al., 2005). Isso se deve ao fato de que, na evolução do homem, as decisões intuitivas o auxiliaram a sobreviver, simplificando seus julgamentos frente a fatos como a necessidade de avaliar riscos rapidamente e lidar com grande quantidade de informações no cotidiano. Assim, a quantidade de informações presente nos problemas decisórios parece estar relacionada à ação dos sistemas 1 e 2 , já que a simplificação de seu processamento (ação do Sistema 1) seria fundamental para a sobrevivência do ser humano. Stanovich e West (2008), em adição, sugerem que o uso de vieses de pensamento (incluindo o efeito de configuração) e habilidades cognitivas não estão necessariamente relacionados, ou seja, características individuais relativas a maiores habilidades não implicam de forma determinante em pensamento racional mais apurado (ou sujeitas a menos vieses).

A quantidade de informações resumidas pode ser considerada como uma descrição não completa dos problemas decisórios, em oposição a descrições expandidas, as quais podem ser entendidas como aquelas que contêm os argumentos completos em relação às alternativas em escolha (Kühberger, 1995). Nessa direção, a quantidade de informações, mesmo que usualmente não relacionada ao funcionamento do Sistema 1 na literatura corrente, parece ter impacto nos resultados das pesquisas sobre o efeito de configuração.

Kühberger (1995), por exemplo, investigou a persistência do efeito de configuração sob risco frente a diferentes quantidades de informação acerca do problema. Para tanto, o autor lançou mão do dilema da doença asiática, utilizado na pesquisa de Tversky e Kahneman (1981), expondo os participantes do estudo a diferentes quantidades de informações (resumida, padrão e expandida). Não foram encontradas diferenças em relação à configuração dos dilemas com a descrição expandida, mas o efeito de configuração usual foi detectado na descrição padrão (utilizada por Tversky \& Kahneman, 1981) e um efeito reverso em relação ao padrão foi encontrado entre os dilemas resumidos. Foi detectado, portanto, o impacto da quantidade de informações dos dilemas no efeito de configuração sob risco.

A observação desses dados parece estar relacionada ao funcionamento do Sistema 1. Kahneman (2003) propôs que o efeito de configuração sob risco poderia ser detectado em função de avaliações intuitivas, já que as pessoas não parecem julgar as alternativas de problemas decisórios com base no conteúdo das alternativas, mas em sua configuração. Por este motivo, a apresentação de duas alternativas idênticas em conteúdo, mas diferentes em configuração, poderiam gerar resultados diferentes. Além disso, conforme evidenciado por Kühberger (1995), o efeito de configuração não foi detectado frente a descrições expandidas dos problemas decisórios utilizados originalmente por Tversky e Kahneman (1981). Assim, o aumento da quantidade de informações poderia estar relacionado à indução de funcionamento do Sistema 2 ou, ainda, a uma espécie de substituição de sua ação, na medida em que se ofereceria o "raciocínio completo" aos sujeitos, evitando a ação da intuição per se.

As explicações para a ocorrência do impacto da quantidade de informações no efeito de configuração são pouco exploradas na literatura, especialmente quando se fala em configuração de objetivos. Com base nesse cenário, este artigo traz os resultados de um experimento que visou mensurar o impacto da quantidade de informações sobre o efeito de configuração de objetivos na tomada de decisão do consumidor. Com isso, pretendeu-se oferecer suporte à idéia de que o efeito de configuração de objetivos pode ser explicado pela ação dos Sistemas 1 e 2. A hipótese é que o efeito de configuração será detectado entre versões resumidas de textos promocionais, em função da ação da intuição (Sistema 1), e não será encontrado nas formas expandidas dos mesmos materiais, devido à ação do raciocínio (Sistema 2).

\section{Método}

O experimento teve como variáveis independentes inter-grupos a quantidade de informações de dois tipos (resumida e expandida) e a configuração de um texto promocional (ganhos e perdas). A variável dependente foi o grau de convencimento do participante em relação ao texto promocional (medida de persuasão).

\section{Participantes}

Participaram do estudo 296 estudantes universitários dos cursos de Comunicação, Administração e Design (141 do sexo masculino e 155 do sexo feminino, com média de idade de 20,74 anos, $D P=3,29)$, designados aleatoriamente a quatro grupos. Dois grupos realizaram o experimento com as versões do texto promocional com o conteúdo resumido, sendo que um deles foi exposto à configuração de ganhos $(n=78)$ e o outro à de perdas $(n=74)$. Os outros dois grupos foram expostos às versões expandidas dos materiais, sendo que um grupo realizou o experimento respondendo à versão de ganhos $(n=70)$ e o outro à versão de perdas $(n=74)$.

\section{Instrumentos}

Foram utilizadas como instrumentos quatro versões de um texto promocional sobre cartões de crédito em meio impresso (configurado em linguagem de ganhos e perdas, com conteúdos expandidos e resumidos). O conteúdo da comunicação foi elaborado a partir de pesquisas realizadas nos sites da Internet das maiores administradoras de cartões de crédito do Brasil, de modo que ele foi moldado de acordo com a realidade do mercado nacional, sem a utilização de características que pudessem identificar qualquer uma das marcas disponíveis. Para inclusão no conteúdo da comunicação, buscou-se privilegiar os pontos de convergência entre as informações apresentadas nos sites das diferentes empresas de cartões de crédito. 
Para a construção das formas resumidas, os conteúdos excluídos das versões expandidas foram os argumentos que visavam sustentar cada tópico do texto promocional. Após a construção das quatro versões do texto promocional (ganhos expandida, ganhos resumida e perdas expandida, perdas resumida), o material foi submetido à avaliação de sete juízes, sendo três profissionais de Marketing, três estudantes universitários e uma lingüista. Os profissionais de Marketing foram indagados acerca da adequação do conteúdo do texto em relação a materiais promocionais dessa natureza, bem como a sua clareza, enquanto que os estudantes foram questionados em relação à quão compreensíveis eram as versões do material. Tanto os profissionais quanto os acadêmicos avaliaram satisfatoriamente o instrumento nos quesitos solicitados, sugerindo pequenos ajustes. A avaliação da lingüista atestou a equivalência em termos da complexidade lingüística e do conteúdo entre as versões do texto promocional, sugerindo algumas adaptações em suas formas. Tendo por base todas as sugestões, foram elaboradas as versões finais do instrumento (Ver Anexo A).

O material-alvo foi apresentado impresso em papel A4, e os participantes foram solicitados a indicar, após a leitura do texto, em folha a parte, o quanto ficaram convencidos com o conteúdo do texto promocional (medida de persuasão). Para a mensuração do grau de convencimento dos participantes em relação ao texto promocional (medida de persuasão), foi utilizada uma escala do tipo Likert de onze pontos (como em estudos similares realizados por Ganzach \& Karsahi, 1995; Ganzach et al., 1997). A escala parte do grau zero e se estende até o dez, sendo ancorada em zero (nada), três (pouco), sete (razoavelmente) e dez (plenamente).

\section{Procedimentos}

Os participantes foram acessados por meio de contatos efetuados com uma universidade particular localizada em Porto Alegre (Rio Grande do Sul, Brasil). Os estudantes participaram de forma voluntária e anônima do estudo, em horário de aula cedido pelo professor.

Todos os participantes receberam, inicialmente, o termo de consentimento livre e esclarecido, elaborado consoante as diretrizes éticas que regulamentam a pesquisa com seres humanos. Aqueles que optaram em participar da pesquisa foram submetidos à apresentação de uma das quatro versões do material-alvo sobre o cartão de crédito, antecedido da instrução que eles receberiam um texto promocional que versava sobre o uso de cartão de crédito.

Após a apresentação do material-alvo, os participantes foram solicitados a indicar o quanto ficaram convencidos com o conteúdo do texto promocional apresentado (medida de persuasão). A única variação nos procedimentos para a coleta dos dados foi o material-alvo apresentado, configurado em ganhos e perdas nas versões resumidas e expandidas, dependendo do grupo ao qual o participante pertencia.

\section{Resultados e Discussão}

Os resultados obtidos através de uma ANOVA apontam para uma diferença nos graus de convencimento em relação às versões do texto promocional entre os grupos pesquisados $[F(3,292)=6,037, p=0,001]$. Uma série de resultados adicionais pode ser visualizada a partir de análises post hoc, cujos resultados são explorados a seguir.

Os dados mostram uma média de convencimento significativamente superior para a versão de ganhos $(M=6,50$, $D P=2,53)$, em comparação com a versão de perdas $(M=5,43, D P=2,53)$, apenas entre as versões resumidas $(p<0,05)$, como pode ser visto na Tabela 1 .

Tabela 1

Médias de Graus de Convencimento e Desvios Padrão entre as Configurações de Ganhos e Perdas das Versões Resumidas e Expandidas

\begin{tabular}{lcccc}
\hline Quantidade de Informações & Configuração & $N$ & Média de Convencimento & $D P$ \\
\hline Resumida & Ganhos & 78 & $6,50^{*}$ & 2,53 \\
& Perdas & 74 & 5,43 & 2,53 \\
Expandida & Ganhos & 70 & 6,84 & 1,95 \\
& Perdas & 74 & 6,82 & 2,17 \\
\hline
\end{tabular}

$* p<0,05$

Esses dados corroboram os resultados de diversos estudos clássicos de configuração sob risco, como os de Tversky e Kahneman (1981), já que a média de convencimento detectada para a versão de ganhos foi superior em comparação à de perdas (entre as formas resumidas). No estudo de Ganzach et al. (1997) sobre configuração de objetivos em mensagens de cartões de crédito, a configuração de ganhos também exerceu efeito persuasivo superior à de perdas no cenário artificial, convergindo com os dados do presente estudo, realizado em salas de aula com um texto fictício. Como Ganzach et al. (1997) propuseram, esses resultados poderiam estar relacionados com o baixo envolvimento com a tarefa e, portanto, com um processamento de informação baseado em informações superficiais ou periféricas.

Explicações adicionais para o menor efeito persuasivo da versão de perdas em relação à de ganhos (em formatos resumidos) podem ser encontradas nas operações do Sis- 
tema 1 e no uso da heurística do afeto. Segundo Kahneman (2003) e Slovic et al. (2002), a heurística do afeto pode ser ativada frente a descrições rotuladas como "negativas" pelos consumidores, gerando menor média de convencimento entre os participantes em função da configuração de perdas. O contrário parece ocorrer na configuração de ganhos, causando avaliações "positivas" sobre o conteúdo da mensagem. Nessa perspectiva, o Sistema 1 seria ativado, já que a avaliação parece não se dar exclusivamente em relação ao conteúdo em si, mas em função do afeto despertados pelas diferentes configurações do mesmo texto promocional (Kahneman, 2003).

Já no que se refere aos problemas com descrição expandida, não foi encontrada uma diferença de médias de convencimento entre as versões de ganhos e perdas $(p=1)$. No estudo aplicado à configuração de objetivos relacionados ao consumo, portanto, os dados sugerem ratificar o fato de que altas quantidades de informação tendem a não produzir o efeito de configuração, em convergência com os dados de Kühberger (1995), o que pode ocorrer devido à ação do raciocínio (Sistema 2) e da conseqüente avaliação de equivalência entre os graus de persuasão causados nos consumidores por ambas as formas expandidas do texto promocional (Kahneman, 2003).

Entre as configurações de ganhos (resumida e expandida), os dados sugerem a inexistência de uma quantidade de informações potencialmente superior para gerar médias de convencimento maiores nos consumidores $(p>0,05)$. As diferentes configurações de ganhos parecem, portanto, ter potenciais de convencimento semelhantes, independente da quantidade de informações. Ambas as versões parecem levar ao rótulo "boa" ou "convincente" em função da ação do Sistema $1 \mathrm{e}$, portanto, da heurística do afeto (Kahneman, 2003; Slovic et al., 2002).

A manipulação da quantidade de informações nas versões de perdas, diferentemente das versões de ganhos, gerou diferenças significativas $(p<0,05)$, com menor grau de convencimento entre os participantes expostos à versão resumida que à expandida. Esse dado sugere que a presença de informações adicionais em materiais de comunicação configurados com linguagem de perdas pode interferir nos julgamentos dos consumidores, elevando seus graus de convencimento a níveis semelhantes aos das versões de ganhos, conforme foi exposto na Tabela 1 .

A diferença dos níveis de persuasão entre as formas resumida e expandida da configuração de perdas ratifica a idéia de que a forma resumida pareceu levar ao uso da heurística do afeto e, portanto, da intuição (Kahneman, 2003; Slovic et al., 2002), já que o texto pareceu ser ta-xado como "pouco convincente". Diferente disso, na versão de perdas com conteúdo expandido, a quantidade de informações pareceu induzir as pessoas à análise, minimizando os efeitos da interferência do afeto "negativo" despertado frente à versão de perdas em forma resumida (Kahneman, 2003).

Os dados do experimento sugerem, portanto, a existência do impacto da quantidade de informações no grau de persuasão da mensagem para o consumidor. A descrição resumida configurada em termos de ganhos e as descrições expandidas (tanto na configuração de ganhos quanto na de perdas) tendem a gerar graus mais elevados de convencimento nos consumidores, em comparação com a versão de perdas em formato resumido. A configuração resumida em termos de perdas deve, portanto, ser cuidadosamente avaliada quando utilizada na comunicação com os consumidores.

\section{Considerações Finais}

Os dados apresentados demonstram a existência do efeito de configuração de objetivos entre formas resumidas de conteúdos de comunicação na tomada de decisão do consumidor. O padrão de respostas encontrado também foi convergente com os estudos prévios sobre o tema (Ganzach \& Karsahi, 1995; Ganzach et al., 1997), que apontaram a configuração positiva como a mais persuasiva em estudos de configuração de objetivos para mensagens de cartões de crédito em cenário artificial, ainda que a literatura em geral sobre o efeito de configuração de objetivos aponte o inverso (maior persuasão para as versões de perdas; Levin et al., 1998).

Os resultados do experimento aqui apresentado, bem como os de Ganzach e seus colaboradores (Ganzach \& Karsahi, 1995; Ganzach et al., 1997), parecem ser divergentes em relação ao padrão esperado de resposta em configuração de objetivos devido à temática dos materiais-alvo apresentados: cartões de crédito. Um maior nível de convencimento foi produzido pela versão de ganhos resumida e, além disso, como proposto por Fagley e Miller (1997), escolhas de risco são menos comuns no domínio dinheiro, o que parece coerente com os resultados apontados.

A quantidade de informações interagiu com a produção do efeito de configuração de objetivos, de modo que ele foi detectado apenas entre versões resumidas dos textos promocionais. Entre as versões resumidas, o efeito de configuração pode ser associado à idéia de que a própria pessoa precisaria julgar ativamente o conteúdo da comunicação, sem informações auxiliares ou pistas, levando-a a um processamento mais automático, intuitivo e emocional, típico do Sistema 1 (Kahneman, 2003). O papel das interferências afetivas (Slovic et al., 2002), portanto, seria relacionado a este processo de economia de tempo e energia nos julgamentos, gerando rótulos positivos para configurações de ganhos e rótulos negativos para configurações que denotem riscos de perdas. Maiores quantidades de informações, nesse sentido, induziriam ao raciocínio (Sistema 2), oferecendo a elaboração da informação em si para as pessoas e minimizando os efeitos associativos e emocionais do processamento simplificado da informação (Kahneman, 2003).

Nesta direção, não seria necessário buscar suporte nas explicações sobre o efeito de configuração sob risco para compreender os fenômenos de configuração de objetivos. A percepção de risco dos consumidores em relação ao 
conteúdo abordado nos texto promocional pareceu uma dimensão apropriada para a discussão dos dados, mas em relação à dimensão risco como sentimento, não como dado concreto (risco como análise), já que a configuração de objetivos não expõe dados probabilísticos sobre as alternativas em foco (Finucane et al., 2000; Peters et al., 2005).

Se a avaliação de risco em relação ao uso de serviços de cartões de crédito aponta segurança em função da configuração positiva de um texto promocional, o efeito de configuração persistiria em uma amostra exclusivamente composta por usuários dos referidos serviços? Poderiam as avaliações sobre textos promocionais ser equivalentes em função de baixo risco percebido em uma amostra composta por usuários desses serviços? A realização de um novo estudo para averiguar a persistência do efeito de configuração de objetivos entre usuários de cartões de crédito seria indicada para elucidar a idéia de que o processo de avaliação sobre o grau de persuasão de mensagens se daria em função do processo intuitivo. Sugere-se avaliar, ainda, o impacto do envolvimento desses consumidores com os serviços de cartões de crédito sobre o efeito de configuração de objetivos.

Em comunicação, recomenda-se que os profissionais utilizem de forma cuidadosa textos promocionais curtos, que parecem levar os consumidores a julgamentos mais intuitivos e, portanto, mais carregados de componentes emocionais e suscetíveis à influência de configuração das mensagens. Nesses casos, no domínio de produtos e serviços relacionados a cartões de crédito, a configuração de ganhos parece ser a mais adequada, mesmo que novos estudos em cenário natural sejam interessantes para averiguar a persistência deste padrão.

Outra questão que merece ser foco de novos estudos é o impacto do envolvimento do consumidor no efeito de configuração de objetivos. Seria interessante avaliar a persistência dos efeitos detectado no experimento apresentado em relação a outros produtos, envolvendo escolhas simples (como fio dental) e complexas (carros e apartamentos), já que a quantidade de esforço cognitivo destinado à escolha poderia interferir na ativação dos sistemas 1 e 2 .

\section{Referências}

Almashat, S., Ayotte, B., Edelstein, B., \& Margrett, J. (2009). Framing effect debiasing in medical decision making. Patient Education and Counseling, 71(1), 102-107.

Bettman, J. R., Luce, M. F., \& Payne, J. W. (1998). Constructive consumer choice processes. Journal of Consumer Research, 25, 187-217.

Epstein, S., Lipson, A., Holstein, C., \& Huh, E. (1992). Irrational reactions to negative outcomes: Evidence of two conceptual systems. Journal of Personality and Social Psychology, 62(2), 328-339.

Fagley, N., \& Miller, P. (1997). Framing effects and arenas of choice: Your money or your life? Organizational Behavior and Human Decision Processes, 71(3), 355-373.

Finucane, M., Alhakami, A., Slovic, P., \& Johnson, S. (2000). The affect heuristic in judgments of risks and benefits. Journal of Behavioral Decision Making, 13, 1-17.
Ganzach, Y., \& Karsahi, N. (1995). Message framing and buying behavior: A field experiment. Journal of Business Research, 32, 11-17.

Ganzach, Y., Weber, Y., \& Bem Or, P. (1997). Message framing and buying behavior: On the difference between artificial and natural environment. Journal of Business Research, 40, 91-95.

Kahneman, D. (2003). A perspective on judgment and choice: Mapping bounded rationality. American Psychologist, 58(9), 697-720.

Kühberger, A. (1995). The framing of decisions: A new look for old problems. Organizational Behavior and Human Decision Processes, 62(2), 230-240.

LeBoeuf, R., \& Shafir, E. (2003). Deep thoughts and shallow frames: On the susceptibility to framing effects. Journal of Behavioral Decision Making, 16, 77-92.

Levin, I., Gaeth, G., Schneider, J., \& Lauriola, M. (2002). A new look at framing effects: Distribution of effect sizes, individual differences, and independence of types of effects. Organizational Behavior and Human Decision Processes, $88(1), 411-429$.

Levin, I., Schneider, J., \& Gaeth, G. (1998). All frames are not created equal: A typology and critical analysis of framing effects. Organizational Behavior and Human Decision Processes, 76(2), 149-188.

Mellers, B. A., Schwartz, A., \& Cooke, A. (1998). Judgment and decision making. Annual Review of Psychology, 4944749477.

Meyerowitz, B. E., \& Chaiken, S. (1987). The effect of message framing on breast self-examination attitudes, intentions, and behavior. Journal of Personality and Social Psychology, 52, 500-510.

Mitchell, V. (1999). Consumer perceived risk: Conceptualizations and models. European Journal of Marketing, 33(12), 163-195.

Mittal, B. (1995). A comparative analysis of four scales of consumer involvement. Psychology and Marketing, 12(7), 663-682.

Payne, J. W., Bettman, J. R., Coupey, E., \& Johnson, E. J. (1992). A constructive process view of decision making: Multiple strategies in judgment and choice. Acta Psychologica, 80, 107-141.

Peters, P., Finucane, M., \& MacGregor, D. (2005). Affect, risk, and decision making. Health Psychology, 24(4), S35-S40.

Slovic, P., Finucane, M., Peters, E., \& MacGregor, D. (2002). The affect heuristic. In T. Gilovich, D. Griffin, \& D. Kahneman (Eds.), Heuristics and biases: The psychology of intuitive judgment (pp. 397-420). New York: Cambridge University Press.

Stanovich, K., \& West, R. (2000). Individual differences in reasoning: Implications for the rationality debate. Behavioral and Brain Sciences, 23, 645-665.

Stanovich, K., \& West, R. (2008). On the relative independence of thinking biases and cognitive ability. Journal of Personality and Social Psychology, 94, 672-695.

Tversky, A., \& Kahneman, D. (1981). The framing of decisions and the psychology of choice. Science, 211, 453-458.

Recebido: 08/06/2009

$1^{a}$ revisão: 14/06/2010

$2^{a}$ revisão: 08/11/2010

Aceite final: $18 / 11 / 2010$ 
Tonetto, L. M. \& Stein, L. M. (2012). Psicologia Cognitiva e Consumo: O Impacto da Quantidade de Informações na Configuração de Objetivos.

\section{Anexo A}

\section{Instrumentos}

A seguir, o leitor encontra as quatro versões do texto promocional, configurado em linguagem de ganhos e perdas, com conteúdos expandidos e resumidos. Vale ressaltar que, quando utilizados para a coleta dos dados, cada frase foi apresentada como um item em separado no texto, utilizando-se alíneas como recurso para dar destaque a cada uma.

Versão de Ganhos Expandida. Utilizando cartão de crédito, você ganha em serviços não acessíveis com o uso de dinheiro ou cheque! Utilizando cartão de crédito, você ganha em segurança, pois não necessita carregar dinheiro ou cheques e não é necessário fornecer dados pessoais a estabelecimentos comerciais durante as compras. Com o uso do cartão de crédito você ganha em conveniência em relação ao uso de dinheiro ou cheque, já que ele garante até 30 dias ou mais para pagar suas compras. Você ganha facilidades quando usa cartão de crédito em relação à utilização de dinheiro e cheque, pois ele ajuda no controle de suas contas, oportunizando a conferência de todas as suas compras. Usando cartão de crédito, você ganha em flexibilidade em relação ao uso de dinheiro ou cheque. Ele aumenta seu poder de compra e você ainda pode parcelar sem a necessidade de aprovação dos estabelecimentos comerciais. Utilizando cartão de crédito, você ganha os seguros não disponibilizados com o uso de dinheiro ou cheque. $\mathrm{O}$ cartão possibilita o uso de seguros contra perda ou furto, seguros de viagens, dentre outros. Ao contrário do que ocorre com a moeda local e os cheques, com o cartão de crédito você pode comprar em todo o mundo. Você realiza compras em supermercados, restaurantes, postos de gasolina, dentre outros, utilizando a maior rede de estabelecimentos credenciados. Com o cartão de crédito, você pode comprar até mesmo pela Internet e pagar na hora com segurança.

Versão de Perdas Expandida. Utilizando dinheiro ou cheque, você perde em serviços acessíveis apenas com o cartão de crédito! Não utilizando o cartão de crédito, você perde em segurança, pois necessita carregar dinheiro ou cheque e fornecer dados pessoais a estabelecimentos comerciais durante as compras. Com o uso de dinheiro ou cheque, você perde em conveniência em relação ao uso do cartão de crédito, já que os estabelecimentos comerciais não garantem 30 dias ou mais para pagar suas compras. Dinheiro e cheque perdem em facilidades em relação ao uso de cartão de crédito, pois não ajudam no controle de suas contas e na conferência de suas compras, como ocorre com o cartão. Usando dinheiro ou cheque, você perde em flexibilidade em relação ao uso do cartão de crédito. Eles não aumentam seu poder de compra como o cartão, e você só pode parcelar com a aprovação dos estabelecimentos comerciais. Utilizando dinheiro ou cheque, você perde os seguros oferecidos pelo cartão de crédito. Dinheiro e cheque não disponibilizam o uso de seguros contra perda ou furto, seguros de viagens, dentre outros. Ao contrário do que ocorre com o cartão de crédito, com a moeda local e os cheques você não pode comprar em todo o mundo. Você pode deixar de comprar em supermercados, restaurantes, postos de gasolina, dentre outros, deixando de utilizar a maior rede de estabelecimentos credenciados. Sem cartão de crédito, você não pode comprar na hora pela Internet com segurança.

Versão de Ganhos Resumida. Utilizando cartão de crédito, você ganha em serviços não acessíveis com o uso de dinheiro ou cheque! Utilizando cartão de crédito, você ganha em segurança em comparação ao uso de dinheiro e cheque. Com o uso do cartão de crédito, você ganha em conveniência em relação à utilização de dinheiro ou cheque. Você ganha facilidades quando usa cartão de crédito em relação à utilização de dinheiro e cheque. Usando cartão de crédito, você ganha em flexibilidade em relação ao uso de dinheiro ou cheque. Utilizando cartão de crédito, você ganha os seguros não disponibilizados com o uso de dinheiro ou cheque. Apenas com o cartão de crédito você pode comprar em todo o mundo, ao contrário do que ocorre com a moeda local e os cheques.

Versão de Perdas Resumida. Utilizando dinheiro ou cheque, você perde em serviços acessíveis apenas com o uso de cartão de crédito! Utilizando dinheiro e cheque, você perde em segurança em comparação ao uso de cartão de crédito. Com o uso de dinheiro ou cheque, você perde em conveniência em relação à utilização do cartão de crédito. Você perde facilidades quando usa dinheiro e cheque em relação à utilização de cartão de crédito. Usando dinheiro ou cheque, você perde em flexibilidade em relação ao uso do cartão de crédito. Utilizando dinheiro ou cheque, você perde os seguros oferecidos apenas com o uso do cartão de crédito. Com a moeda local e os cheques você não pode comprar em todo o mundo, ao contrário do que ocorre com o cartão de crédito. 